\title{
« La guerre d'Algérie dans les mémoires française et algérienne »
}

Conférence de Benjamin STORA

Juliette Guilbaud et Rodolphe Keller

\section{(2) OpenEdition}

\section{Journals}

Édition électronique

URL : http://journals.openedition.org/ifha/128

DOI : $10.4000 /$ ifha. 128

ISSN : 2198-8943

Éditeur

IFRA - Institut franco-allemand (sciences historiques et sociales)

Édition imprimée

Date de publication : 6 février 2011

Pagination : $50-53$

ISSN : 2190-0078

Référence électronique

Juliette Guilbaud et Rodolphe Keller, « «La guerre d'Algérie dans les mémoires française et algérienne » », Revue de l'IFHA [En ligne], 3 | 2011, mis en ligne le 01 octobre 2012, consulté le 03 mai 2019. URL : http://journals.openedition.org/ifha/128; DOI : 10.4000/ifha.128

Ce document a été généré automatiquement le 3 mai 2019.

(CIFHA 


\title{
«La guerre d'Algérie dans les mémoires française et algérienne »
}

\author{
Conférence de Benjamin STORA \\ Juliette Guilbaud et Rodolphe Keller
}

\section{NOTE DE L'ÉDITEUR}

Francfort-sur-le-Main, 1er février 2011

Le 1er février 2011, face à une salle bondée du campus de Westend, à Francfort-sur-leMain, Benjamin STORA (univ. Paris XIII/Inalco), spécialiste du nationalisme algérien et de la guerre d'Algérie (1954-1962), tenait une conférence sur une thématique sensible, qui a pris une ampleur considérable en France depuis quelques années : celle de la mémoire ou plutôt des mémoires - autour de la guerre d'indépendance algérienne, conflit sanglant suite auquel la France perdait sa plus ancienne colonie africaine. B. STORA articula son intervention sur la complexité des enjeux mémoriels, sur l'affrontement entre des mémoires concurrentes, qui expriment des sensibilités et des lectures des événements franchement opposées. Différents groupes mobilisent leur propre vécu des drames de la guerre, dans le cadre de revendications mémorielles qui, difficilement conciliables, semblent devoir s'exclure mutuellement.

C'est surtout depuis une dizaine d'années que ce besoin de mémoire s'est affirmé. Plusieurs séries d'événements doivent être pris en compte. En juin 1999, l'Assemblée nationale adoptait la décision de nommer « guerre d'Algérie » ce qui n'était auparavant qu'une "opération de maintien de la paix ». Ce changement politique s'accompagna de l'apparition de nouveaux témoignages, par d'anciens insurgés algériens, par d'anciens combattants, qui tantôt exprimèrent de la repentance, tantôt revendiquèrent des crimes perpétrés pendant la guerre. Cette tension mémorielle résulta également d'un renouveau historiographique lié à l'ouverture d'archives permettant de nouvelles recherches, comme la thèse de Raphaëlle Branche sur la torture par l'armée française. Plusieurs événements, donc, venant soutenir un mouvement de fond plus général : une tendance de 
plus en plus marquée au retour de mémoires douloureuses, comme en Allemagne à propos des crimes commis pendant la Seconde Guerre mondiale, en Israël concernant la relecture par une nouvelle génération d'historiens des événements fondateurs de la société israélienne, ou encore aux États-Unis au sujet de la guerre du Vietnam. Ce fut l'occasion pour B. STORA de confronter les situations française et algérienne, où les enjeux se sont toujours posés de manière très différente.

En France, cette guerre des mémoires est l'expression de groupes pour lesquels les drames de la guerre d'Algérie constituent un facteur d'identité d'autant plus significatif qu'ils constituent des minorités (harkis, pieds-noirs, immigrés algériens...). Elle prend la forme de revendications concurrentes, qui se sont amplifiées surtout depuis la fin des années 1990. Dans une inflation des propositions commémoratives, chaque groupe se mobilise autour de l'événement qui cristallise ses griefs: les pieds-noirs (Français d'origine européenne, installés en Afrique du Nord jusqu'à l'époque de l'indépendance) se rassemblent autour de la date du 26 mars 1962, qui vit l'armée française réprimer dans le sang une manifestation de l'Organisation armée secrète (OAS) à Alger. D'autres proposent de commémorer le 19 mars 1962, date des accords d'Évian, qui mit un terme à la guerre. B. STORA souligna l'impossibilité pour un pays de construire sa mémoire autour d'une défaite militaire. Il est ainsi impossible d'accorder les acteurs sur une date, car les positions sont largement irréconciliables. Alors que certains peinent aujourd'hui encore à accepter la perte de l'Algérie, aucun personnage ou événement historique ne peut faire l'unanimité et rassembler. En février 2005, le vote par l'Assemblée nationale d'une loi rappelant le « rôle positif de la colonisation ", sous l'influence de nostalgiques de l'Algérie française, ne faisait que crisper plus encore les positions.

En Algérie, la question se pose très différemment : les Algériens n'ont que faire des états d'âme des Français concernant la guerre. Le conflit est ici une "guerre d'indépendance " et représente une victoire, un événement fondateur. Après la guerre, le Front de libération nationale (FLN), auréolé de sa victoire, se constituait en parti unique et s'emparait de manière exclusive du pouvoir. L'État issu de l'indépendance s'appropria alors la construction mémorielle et l'instrumentalisa en vue d'asseoir sa propre légitimité. Il passa ainsi sous silence les conflits qui opposèrent les nationalistes algériens pendant ou après la guerre, comme les meurtres entre nationalistes ou le massacre de plus de trois cents villageois à Melouza par le FLN, en mai 1957. Récemment, en Algérie aussi, s'observe un retour mémoriel qui se traduit par la réhabilitation de certains noms les « pères maudits » du nationalisme algérien - comme Messali Hadj ou Abane Ramdane, tous deux tués par le FLN dans le cadre de conflits algéro-algériens. Les jeunes générations algériennes mettent aujourd'hui en cause la récupération politique de la mémoire de l'indépendance par l'État algérien. Elles revendiquent une évolution démocratique, qui passe par une relecture de la mémoire de la guerre monopolisée par le FLN. Le processus mémoriel doit alors, pour certains, se libérer de l'appropriation politique qui en a été faite, pour servir de tremplin à un changement démocratique.

Cette dernière réflexion permit à $\mathrm{B}$. STORA de faire le lien avec les événements qui sont intervenus dans le monde arabe depuis décembre 2010, après que la révolte tunisienne contre le président Ben Ali eut marqué le début de ce que certains baptisèrent le «Printemps des peuples arabes ». B. STORA proposa sa propre analyse des événements, qu'il replaça dans une perspective historique. Il y voit l'expression d'une revendication démocratique, qui traduit une évolution à long terme et fait suite à l'échec des principaux courants politiques des pays arabes après l'indépendance, comme l'islamisme politique ou 
le nationalisme arabe, auquel se rattachent nombre de régimes autoritaires organisés en partis uniques (le FLN en Algérie, le parti Ba'as en Syrie et en Irak) - régimes qui, précisément, ont le plus souvent fondé leur légitimité et leur domination politique sur une appropriation de l'héritage de l'indépendance.

Après cette présentation, qui révéla les talents d'improvisateur d'un B. STORA préférant rapidement s'affranchir du cadre contraignant de ses notes, la place était donnée au jeu des questions-réponses, auquel l'orateur se prêta volontiers. Le public, à la fois divers et très intéressé, constitué en partie de personnes directement concernées par les sujets évoqués, revint sur plusieurs points.

Une première question porta précisément sur le rapprochement que l'on pourrait faire ou non - entre la guerre d'Algérie et la situation récente en Tunisie, parfois appelée « révolution de Jasmin ». En ce qui concerne l'Algérie, le terme de "révolution » a été utilisé du côté algérien après 1962, en concurrence avec ceux de "guerre de libération nationale » et de "guerre d'indépendance ", ce dernier étant toutefois plus récent et le plus utilisé en Algérie aujourd'hui. Et B. STORA de souligner un affaiblissement du terme de "révolution ", qui renverrait aujourd'hui plutôt à des bouleversements à venir, car le problème de la démocratie politique demeure encore largement à résoudre. De la même façon, on peut considérer que le terme de « révolution » employé à propos de la Tunisie aujourd'hui renvoie au démarrage d'un processus de démocratisation de la vie politique.

Interrogé sur le rôle de l'État, en France, dans le processus mémoriel (voir la polémique sur le prétendu «rôle positif de la colonisation»), B. STORA répondit à une autre question, celle de l'engagement des historiens dans le débat politique: lui-même reconnait son engagement, respectant toutefois le retrait de certains historiens hors du champ politique.

Furent aussi évoqués lors de la discussion les traumatismes suscités côté français par la guerre d'Algérie (outre les difficultés rencontrées tantôt par les pieds-noirs, tantôt par les harkis, l'incompréhension devant le rôle de la gauche à l'époque et celui d'un certain nombre de pays étrangers ayant soutenu l'indépendance de l'Algérie). Une dernière intervenante souleva enfin le problème de l'unité algérienne aujourd'hui: B. STORA préfère pour sa part parler d'un ensemble de singularités, dont le maintien est mis en péril depuis l'indépendance. 